\title{
ARTICLE
}

\section{Inferring personal economic status from social network location}

\author{
Shaojun Luo, Flaviano Morone, Carlos Sarraute ${ }^{2}$, Matías Travizano² \& Hernán A. Makse
}

It is commonly believed that patterns of social ties affect individuals' economic status. Here we translate this concept into an operational definition at the network level, which allows us to infer the economic well-being of individuals through a measure of their location and influence in the social network. We analyse two large-scale sources: telecommunications and financial data of a whole country's population. Our results show that an individual's location, measured as the optimal collective influence to the structural integrity of the social network, is highly correlated with personal economic status. The observed social network patterns of influence mimic the patterns of economic inequality. For pragmatic use and validation, we carry out a marketing campaign that shows a threefold increase in response rate by targeting individuals identified by our social network metrics as compared to random targeting. Our strategy can also be useful in maximizing the effects of large-scale economic stimulus policies.

\footnotetext{
${ }^{1}$ Levich Institute and Department of Physics, City College of New York, New York, New York 10031, USA. ${ }^{2}$ Grandata Labs, 550 15th Street, San Francisco, California 94103, USA. Correspondence and requests for materials should be addressed to H.A.M. (email: hmakse@lev.ccny.cuny.edu).
} 
T he long-standing problem of how the network of social contacts ${ }^{1-3}$ influences the economic status of individuals has drawn large attention due to its importance in a diversity of socioeconomic issues ranging from policy to marketing ${ }^{4-7}$. Theoretical analyses have pointed to the importance of the social network in economic life ${ }^{5}$ as a medium to diffuse ideas ${ }^{8,9}$ through the effects of 'structural holes' ${ }^{10}$ and 'weak ties' in the network ${ }^{4}$. Likewise, research has recognized the positive economic effect of expanding an individual's contacts outside its own tightly connected social group ${ }^{1,11-13}$. While previous work has established the importance of social network influence to economic status, the problem of how to quantify such correspondence via social network centralities or metrics ${ }^{3,14}$ remains open.

Studies employing mobile phone communication data and other social indicators have found a variety of network effects on socioeconomic indicators such as job opportunities ${ }^{15,16}$, social mobility ${ }^{17-19}$, economic development ${ }^{6,20-22}$ and consumer behaviour ${ }^{23,24}$. Recent work also provides evidence of such effects on an individual's wealth, and highlights the need for better indicators ${ }^{25}$. Recently, a numerical study has tested the effect of network diversity on economic development ${ }^{6}$. This study analysed economic development defined at the community level. However, the question of how social network metrics may be used to infer financial status at the individual level-necessary, for instance, for micro-target marketing or social intervention campaigns-still remains unanswered. The difficulty arises, in part, due to the lack of empirical data combining an individual's financial information with the pattern of their social ties at the large-scale network level of the whole society.

In this work, we address this problem directly by combining two massively large data sets: a social network of the whole population of a Latin American country and financial banking data at the individual level. We discover that the optimality of an individual's location in the network, which is measured by the collective influence (CI) metric ${ }^{26}$, is highly correlated with the individual's economic status at the population level: the larger the $\mathrm{CI}$, the higher the socioeconomic level. The goodness of fit of this correlation can be as high as $R^{2}=0.99$ when age is also included. These results indicate that the location's optimality in the social network measured by the CI metric can accurately predict socioeconomic indicators at the personal level.

The top $1 \%$ of the economic stratum has precise network patterns of ties formation showing relatively low local connectivity surrounded by a hierarchy of hubs strategically located in spheres of influence of increasing size in the network. Such a pattern is not observed in the rest of the population, in particular, in the bottom $10 \%$ characterized by low values of CI. Thus, the influence measured from social network patterns mimics the inequality observed in economic status ${ }^{27}$.

We also find a high correlation between the link diversity of individuals and their financial status $\left(R^{2}=0.96\right)$, employing the analysis based on network location and age. Analysis of the covariance suggests that the effect of network influence is significant and independent from other factors. We validate these results by carrying out a targeted marketing campaign in which we compare the response rate for different groups of people with different network locations. By targeting the group with the top CI values, the response rate can reach as high as $1 \%$; approximately three times the response rate found by random targeting and five times the response rate of the low CI people.

Thus, individuals with high socioeconomic status (top 1\%) develop a very characteristic pattern of social ties as compared to the bottom $10 \%$. While this result may be expected, it is remarkable that the difference in pattern of social interactions between the rich and the poor can be precisely captured by a network metric measuring their $\mathrm{CI}$ in the social network ${ }^{26}$.
The top socioeconomic layer of society also represents the minimal set of people that provides integrity to the whole social network through their large CI. The fact that individuals of higher economic status are located in regions of large $\mathrm{CI}$ in the network elevates previous anecdotal evidence to a principle of network organization through the optimization of influence of affluent people affecting the structural integrity of the social network. At the same time, it suggests the emergence of the phenomenon of CI in society as the result of the optimization of socioeconomic interactions.

\section{Results}

Network construction. The social network is constructed from mobile (calls and SMS metadata) and residential communications data collected for a period of 122 days (Supplementary Note 1, aggregated data at kcorelab.com). The database contains $1.10 \times 10^{8}$ phone users. After filtering the non-human active nodes by a machine-learned model trained on human natural communication behaviour (Supplementary Note 2, with Supplementary Figs 1-4), we construct a final network of $1.07 \times 10^{8}$ nodes in a giant connected component made of $2.46 \times 10^{8}$ links. The ties, or links, in the network correspond to phone call communications, since we expect that communication patterns are indicative of an individual's location in the social network $^{28-30}$. The financial cost of using phone services makes it possible that there is a systematic bias in how much wealthy individuals use the phone services relative to people that have less money to spend on phone calls. Although the effect might be limited (Supplementary Note 1), we cannot rule out this possibility with the present data.

Financial status is obtained from the combined credit limit on credit cards assigned by banking institutions to each client. The credit limit is based on composite factors of income and credit history and therefore reflects the financial status of the individual (see discussion in Supplementary Note 1). The credit limit is pulled from an encrypted bank database and identified by the encrypted clients' phone numbers registered in the bank. Thus, we are able to precisely cross-correlate the financial information of an individual with their social location in the phone call network at the country level. There are $5.02 \times 10^{5}$ bank clients who have been identified in the mobile network whose credit limit ranges from USD $\$ 50$ to $\$ 3.5 \times 10^{5}$ (converted from the country of study). Thus, the data sets are precisely connected providing an unprecedented opportunity to test the correlation between network location and financial status.

Despite the large scale of our data source, we note that working on a single specific country as in the present study is not enough to grant generality to our results. To test the general validity of the present results, access to other countries' whole-populationlevel communication and banking data sets would be needed. As more data sets become available, the generality of our results can be tested across different economic and social systems.

Figure 1a,b shows the communication patterns geolocalized across the country of individuals in the top $1 \%$ and bottom $10 \%$ of credit limits, respectively. The inequality in the patterns of communication between the top economic class and the lowest is striking and mimics the economic inequality at the country level ${ }^{27}$. It is visually apparent that the top $1 \%$ (accounting for $45.2 \%$ of the total credit in the country) displays a completely different pattern of communication than the bottom $10 \%$; the former is characterized by more active and diverse links, especially connecting remote locations and communicating with other equally affluent people. Further results using entropy analysis also suggest that the network structure may be significantly different between the people in the top and bottom 


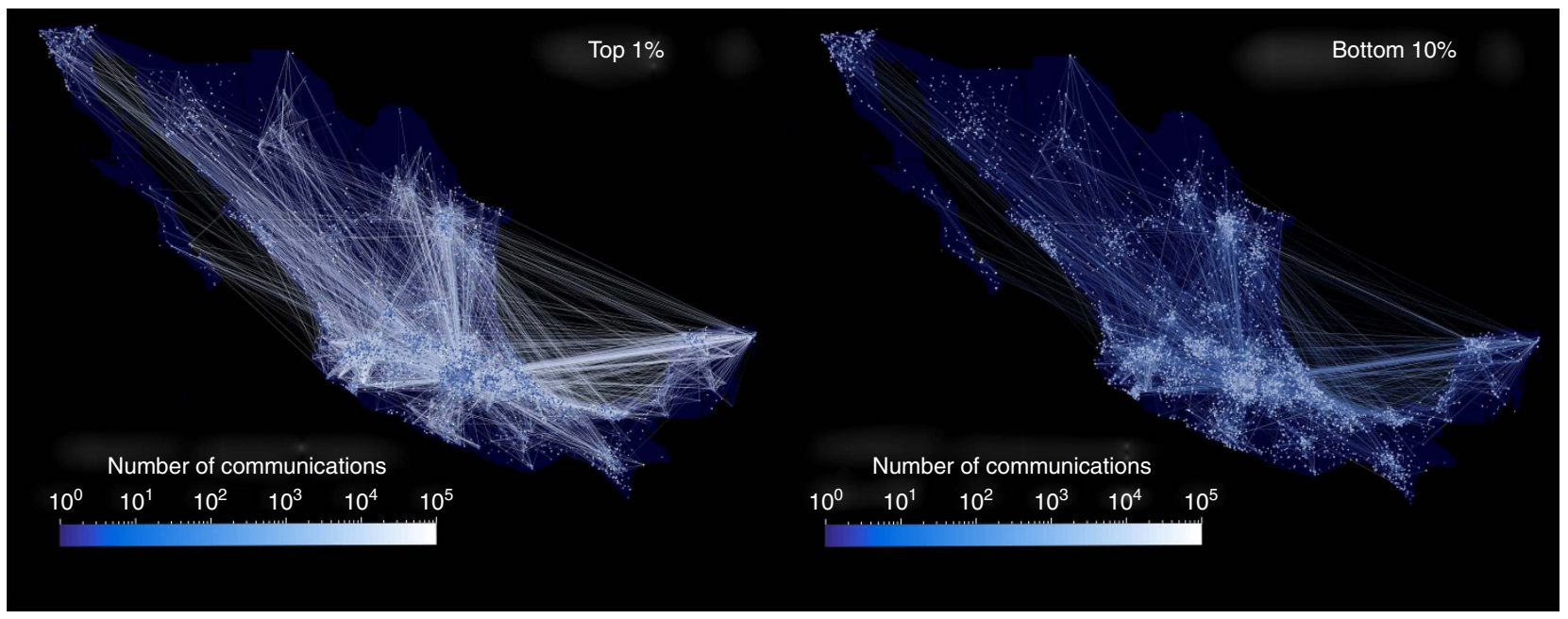

C

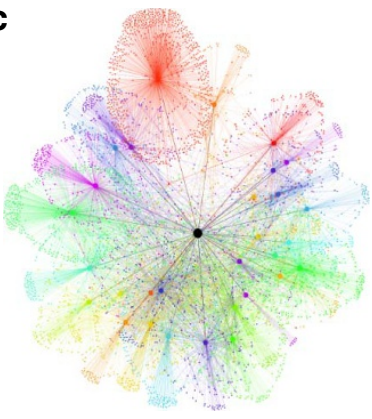

d

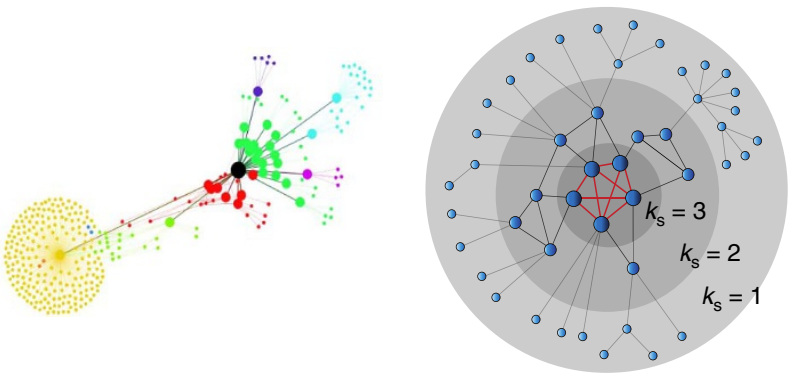

f

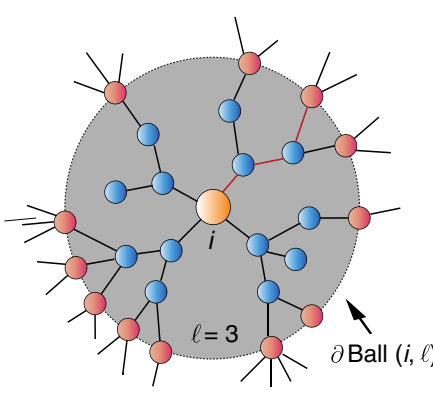

Figure 1 | Patterns of network influence mimic patterns of income inequality. Visualization of communication activity of the population in a the top $1 \%$ (with credit limit larger than USD $\$ 25,000$, converted, in the country of study) and (b) bottom $10 \%$ (with credit limit smaller than USD $\$ 600$, converted) of total credit limit classes. Links are between bank clients who have registered their zip code. Resolution of both plots is $1,700 \times 1,000$. The number of bank clients inside each community is reflected by the size of the node. Average credit limit is denoted by a node's grayscale. The colour and thickness of the edges reflects the number of communication events between different communities. (c) Examples of the ego-network (extended to two layers) for an individual in the top $1 \%$ wealthy class and (d) an individual in the bottom $10 \%$ class. The networks show two distinct patterns of social ties according to high and low economic status: the former is characterized by large $\mathrm{Cl}$, the latter by low $\mathrm{Cl}$. (e) Schematic representation of a network under k-shell decomposition ${ }^{33}$. (f) Example of the calculation of $\mathrm{Cl}$. The $\mathrm{Cl}$ Ball $(i, \ell)$ of radius $\ell=3$ around node $i$ is the set of nodes contained inside the sphere and $\partial \mathrm{Ball}$ is the set of nodes on the boundary (brown). $\mathrm{Cl}$ is the degree-minus-one of the central node times the sum of the degree-minus-one of the nodes at the boundary of the sphere of influence.

quantile rankings of credit limit (Supplementary Note 3; Supplementary Table 1). Particular examples of the extended egonetworks for two individuals (with same number of ties) ranking in the top $1 \%$ and bottom $10 \%$ provide a zoomed-in picture of such differences (Fig. 1c,d, respectively). The wealthiest 1-percenters have higher diversity in mobile contacts and are centrally located, surrounded by other highly connected people (network hubs). On the other hand, the poorest individuals have low contact diversity and are weakly connected to fewer hubs. The crux of the matter is to find a reliable social network metric to quantify this visual difference in the patterns of network structure between the rich and the poor, as we show next.

Network influence and financial status. Many metrics or centralities have been considered to characterize the influence or importance of nodes in a network ${ }^{3,14,31}$. Here we consider only those centralities that can be scaled up to the large network size considered here (Fig. 1e,f; Supplementary Note 4): (a) degree centrality $k_{i}$ (number of ties of individual $i$ ) is one of the simplest ${ }^{3}$, (b) PageRank, of Google fame ${ }^{32}$, is an eigenvector centrality that includes the importance of not only the degree, but also the nearest neighbors, (c) the k-shell index $k_{\mathrm{s}}$ of a node (Fig. 1e), that is, the location of the shell obtained by iteratively pruning all nodes with degree $k<k_{\mathrm{s}}$ (ref. 33), and (d) the CI of a node with degree $k_{i}$ (Fig. 1f) in a sphere of influence of size $\ell$ defined by the frontier of the influence ball $\partial \operatorname{Ball}(i, \ell)$, and predicted to be $\mathrm{CI}=\left(k_{i}-1\right) \sum_{j \in \partial \operatorname{Ball}(i, \ell)}\left(k_{j}-1\right)$ by optimal percolation theory ${ }^{26}$. As opposed to the other heuristic centralities, CI is derived from the theory of maximization of influence in the network ${ }^{34}$. The top CI nodes are thus identified as top influencers or superspreaders of information, and they are so by positioning themselves at strategic locations at the centre of spheres surrounded by hubs hierarchically placed at distances $\ell$ (Fig. 1d). These collective influencers also constitute an optimal set that provides integrity to the social fabric: they are the smallest number of people that, upon leaving the network (a process mathematically known as optimal percolation ${ }^{26}$ ), would disintegrate the network into small disconnected pieces.

By definition, all of the metrics have similarities (for example, they are proportional to $k$, and PageRank and CI are based on the largest eigenvalues of the adjacency and non-backtracking matrices, respectively ${ }^{26}$ ), and indeed, we find that their values in the phone communications network are correlated (Suppleme- 

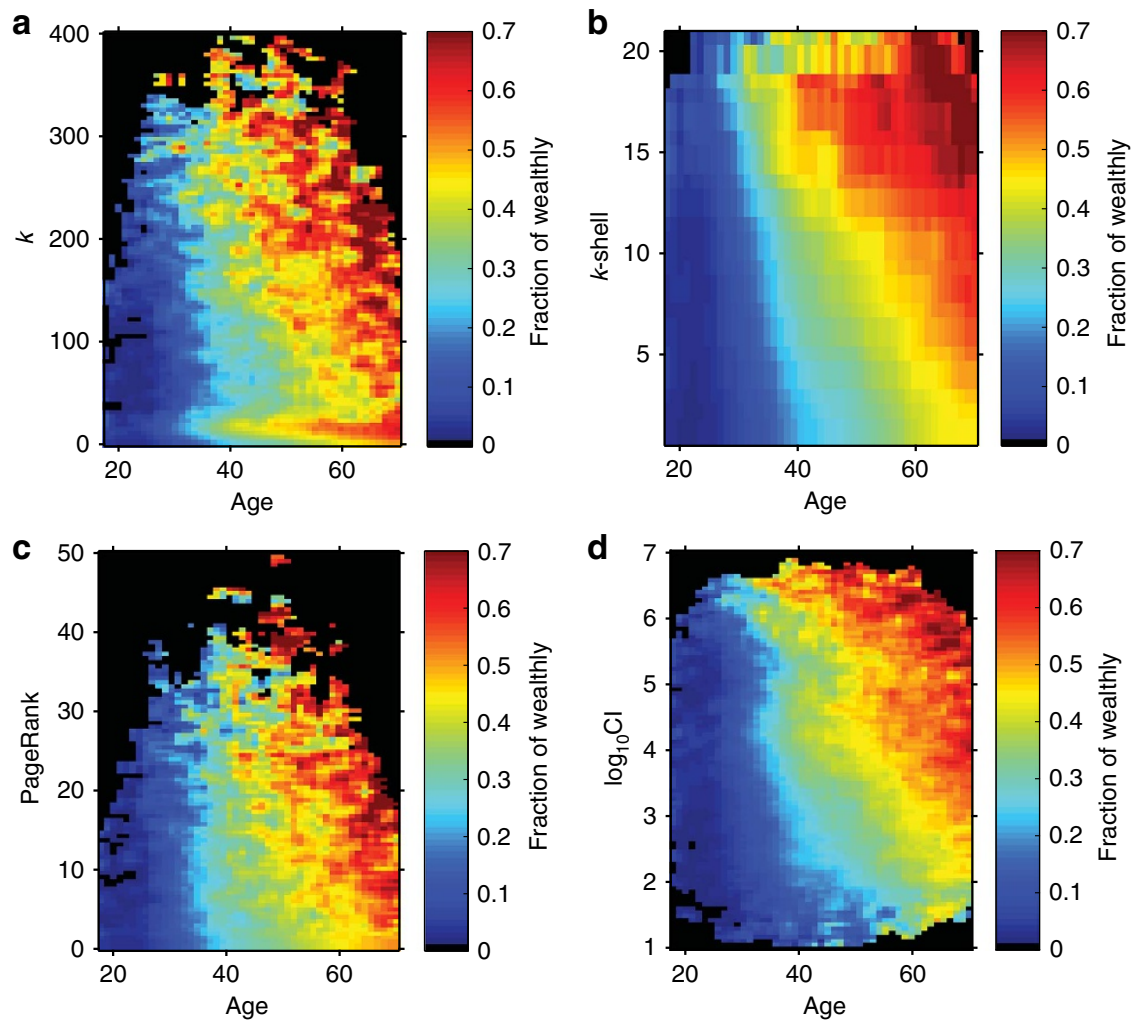

Figure 2 | Fraction of wealthy individuals versus age and network metrics. Correlation between the fraction of wealthy individuals versus age and (a) degree $k\left(R^{2}=0.92\right)$, (b) k-shell $\left(R^{2}=0.96\right)$, (c) PageRank $\left(R^{2}=0.96\right)$ and $(\mathbf{d}) \log _{10} \mathrm{Cl}\left(R^{2}=0.93\right)$. Only those groups with population $>20$ are shown in the plot. The four metrics correlate well with financial status when considered with age. Further correlations are studied in Supplementary Note 6 , indicating that $\mathrm{Cl}$ could be considered as the most convenient metric out of the four due to its high resolution.

ntary Table 2). More interestingly, Fig. 2 provides evidence of correlation of the four network metrics with financial status (ranked credit limit) when we control for age, indicating that the network location correlates with financial status. In this figure, we plot the fraction of wealthy individuals (defined as top 4th quantile, equivalent to a credit limit greater than USD $\$ 4,000$; see Supplementary Note 5 for details about validation methods and ref. 30) in a sampling grid for a given value of age and social metric as indicated.

While all of the social metrics show correlations with financial status when considered with age (Fig. 2), the question remains of which metric is the most efficient predictor. Strong correlations with economic wellness are observed for the feature pairs (age, k-shell; $R^{2}=0.96$, Fig. 2b) and (age, CI; $R^{2}=0.93$, Fig. 2d). Supplementary Note 6 (Supplementary Figs 7-9) provides further comparison when considering the metrics alone, indicating that $\mathrm{k}$-shell and $\mathrm{CI}$ better capture the correlation with credit limit. Between these two metrics, CI guarantees a requirement for both strong correlation and sufficient resolution. K-shell cannot capture further details due to its limitation of values ( $\mathrm{k}$-shell ranges from 1 to 23 , dividing the whole population into this small number of shells with a typical shell containing tens of millions of people), while CI spans over seven orders of magnitude; see Supplementary Fig. 5. This high resolution implies that $\mathrm{CI}$ is a more accurate social signature for the financial status of the individuals. According to its definition (Fig. 1d), a top CI node is a moderate-to-strong hub surrounded by other hubs hierarchically placed at distance $\ell$. However, we emphasize that CI is just a useful strategy for the reasons shown above, and by no means the only or best strategy to correlate the wealth of individuals and their network influence.
While the theory behind $\mathrm{CI}$ is a global maximization of influence, CI represents the local approximation to this global optimization. Thus, CI represents a balance between a global optimization and its local approximation, taking into account the first 2 or 3 layers of neighbours via the parameter $\ell$, which represents the size of the sphere of influence used to define the importance of a node, Fig. 1d. By changing $\ell$, we discover that CI with $\ell=2$ is sufficient to capture the correlation between network influence and wealth (Supplementary Fig. 10).

To track the effect of CI independently of age, we investigate the effects of CI inside two specific age groups in Fig. 3a,b. In both age groups, high CI is always accompanied by a higher population of wealthy people. A relatively smaller slope in age group $<30$ suggests that the CI network effect is more sensitive for older people with more mature and stable economic levels, than for younger people (Supplementary Fig. 6). When we combine age and $\mathrm{CI}$ quantile ranking into an age-network composite: $\mathrm{ANC}=\alpha$ Age $+(1-\alpha) \mathrm{CI}$, with $\alpha=0.5$, a remarkable correlation $\left(R^{2}=0.99\right.$, Fig. 3c) is achieved. By combining network information with age, the probability to identify individuals with a high credit limit reaches $\sim 70 \%$ at the highest earner level. Such a level of accuracy renders the model practical to infer individuals' financial fitness using network CI as we show next.

Validation by marketing campaign. To validate our strategy, we perform a social marketing campaign whose objective is the acquisition of new credit card clients, by sending messages to affluent individuals (as identified by their CI values) and inviting the recipients to initiate a product request (Supplementary Note 8). We note that in this experiment we use an independent data set from a different time frame, and we use only the CI 
a

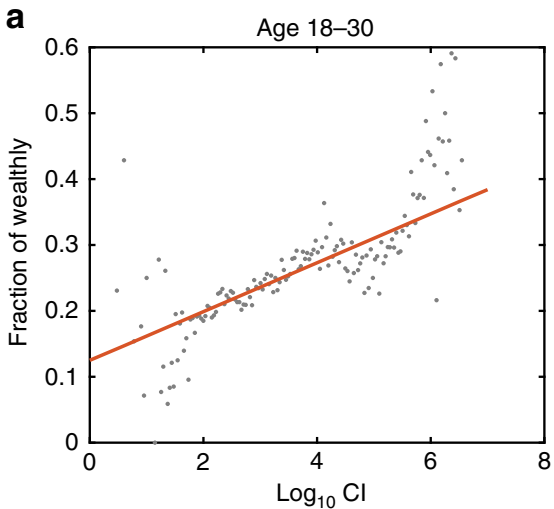

c

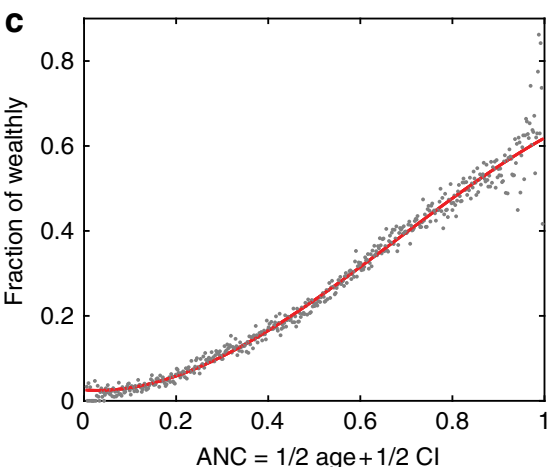

b
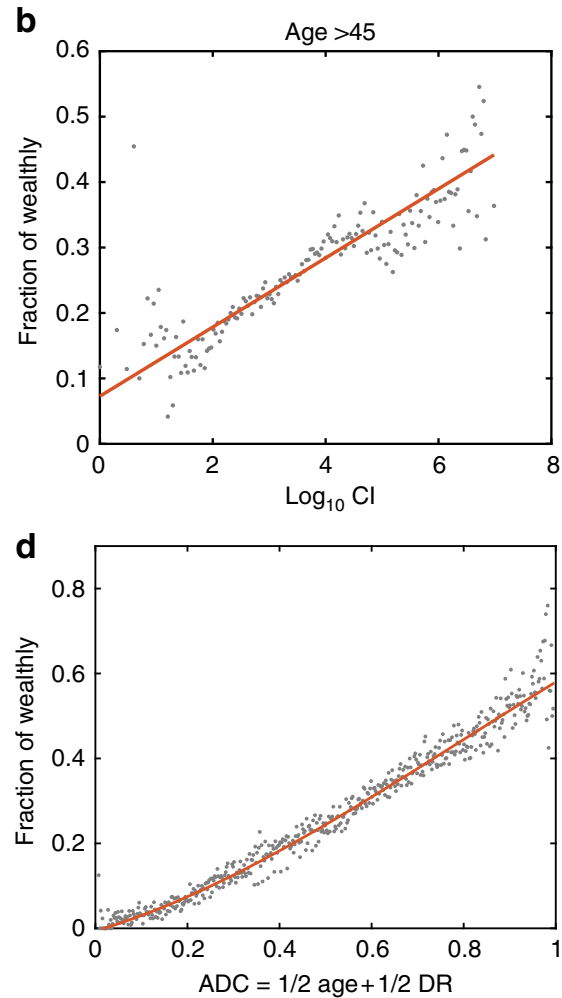

Figure 3 | Fraction of wealthy individuals over different age and composite ranking groups. Correlation between the fraction of wealthy individuals as given by the top $25 \%$ credit limit and $\mathrm{Cl}$ in different age groups of (a) 18-30 and (b) $>45$. Correlations between top economic status and large $\mathrm{Cl}$ as determined by $\mathrm{Cl}$ values in different ages are significant in all age groups, while the slope of the linear regression is larger in the older group (0.053 compared to 0.037). (c) Age-network composite ranking $\mathrm{ANC}=1 / 2 \mathrm{Age}+1 / 2 \mathrm{Cl}$, and (d) age-diversity composite ranking $\mathrm{ADC}=1 / 2 \mathrm{Age}+1 / 2 \mathrm{DR}$. By combining the network metrics with age into a composite index, the chance to identify people of high financial status reaches $\sim 70 \%$ for high values of the composite. Both $R^{2}$ s show a high level of correlation $\left(R^{2}=0.99\right.$ and 0.96 for ANC and ADC, respectively), making both composites good predictors of wealth in practical applications.

Table 1 | Results of the real-life marketing campaign.

\begin{tabular}{|c|c|c|c|c|}
\hline $\mathrm{Cl}$ range & Count & Quantile & Answered yes & Response rate \\
\hline$(0,48)$ & 66,495 & 0.1 & 170 & $0.26 \%$ \\
\hline$(48,246)$ & 65,164 & 0.2 & 218 & $0.33 \%$ \\
\hline$(246,600)$ & 65,961 & 0.3 & 316 & $0.48 \%$ \\
\hline$(600,1,144)$ & 65,376 & 0.4 & 332 & $0.51 \%$ \\
\hline$(1,144,1,992)$ & 65,477 & 0.5 & 363 & $0.55 \%$ \\
\hline$(1,992,3,408)$ & 65,477 & 0.6 & 458 & $0.70 \%$ \\
\hline$(3,408,6,032)$ & 65,736 & 0.7 & 493 & $0.75 \%$ \\
\hline$(6,032,11,772)$ & 65,641 & 0.8 & 555 & $0.8 \%$ \\
\hline$(11,772,28,740)$ & 65,683 & 0.9 & 657 & $1.0 \%$ \\
\hline$(28,740,2,719,354)$ & 65,683 & 1.0 & 573 & $0.87 \%$ \\
\hline
\end{tabular}

values extracted from the network to classify the targeted people. Specifically, we use the communications network resulting from the aggregation of calls and SMS exchanged between users over a period of 91 days. The resulting social network contains $7.19 \times 10^{7}$ people and $3.51 \times 10^{8}$ links. The campaign was conducted on a total of 656,944 people who were targeted by an SMS message offering the product according to their CI values in the social network. We also sent messages to a control group of 48,000 people, chosen randomly. To evaluate the campaign, we measured the response rate, that is, the number of recipients who requested the product divided by the number of targeted people, as a function of CI. In the control group, the response rate to the messages was $0.331 \%$. Our results show that groups of increasing CI show an increase in their response rate, with a sound threefold gain in the rate of response of the top influencers (as identified by top CI values) when compared to the random case. When we compare the response of the high CI to the lowest CI people, the response rate increase fivefold. The results of the experiment are summarized in Table 1 and Fig. 4.

Analysis of covariance. We note that our validation is indirect since it is not a direct prediction of financial status, but a rate of successful response to a marketing campaign. This success 


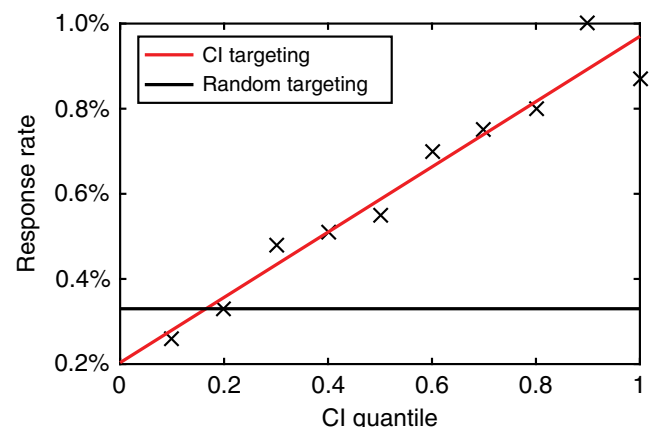

Figure 4 | Response rate versus $\mathrm{Cl}$ quantile in the real-life $\mathrm{Cl}$-targeted marketing campaign. The response rate increases approximately linearly with $\mathrm{Cl}$ ranking. The $\mathrm{Cl}$-targeted campaign shows a threefold gain for the top influencers with high $\mathrm{Cl}$, as compared with a campaign targeting a randomized control group.

rate may actually depend on a number of other factors that may correlate with the network centrality. Thus, the CI metric may not necessarily be the only cause of the success rate of the targeted campaign (for instance, geographical location may be also important). To address this point, we perform an analysis of covariance ${ }^{35}$ on all of the features to which we have access (age, gender and registered zip code) to test the variance caused by the network metrics and other factors (details in Supplementary Note 5 and Supplementary Table 3). Analysis of covariance shows that the effects of the network metrics are independent from those of the other factors. The correlation between the CI and the fraction of wealthy people is positive and significant $(P<0.001)$ in all groups of geographical communities, across genders, and among all age groups older than 24 years (Supplementary Fig. 6). The same significant results are also obtained under different thresholds of wealth. Such significant and robust network effects imply that network metrics may be a potential indicator for financial status.

Network diversity and financial status. Our combined data sets also offer the possibility to test the importance of the diversity of links, as measured by ties to distant communities in the network not directly connected to an individual's own community, at the level of single individuals ${ }^{4-6}$. To this end, we first detect the communities in the social network by applying fast fold modularity detection algorithms (Supplementary Note 7; Supplementary Fig. 11) ${ }^{36,37}$. The diversity of an individual's links can be quantified through the diversity ratio $\mathrm{DR}=W_{\text {out }} / W_{\text {in }}$ (ref. 10), defined as the ratio of total communication events with people outside their own community, $W_{\text {out }}$, to those inside their own community, $W_{\text {in }}$. This ratio is weakly correlated to $\mathrm{CI}(R=0.4)$, suggesting that it captures a different feature of network influence. We implement the same statistics of composite ranking as before, resulting in an age-diversity composite $\mathrm{ADC}=\alpha$ Age $+(1-\alpha) \mathrm{DR}$, with weight $\alpha=0.5$. The result (Fig. 3d) shows that ADC correlates with individual financial well-being, generalizing the aggregated results in ref. 6 to the individual level. Thus, the social metrics considered, DR and $\mathrm{CI}$, express the fact that higher economic levels are correlated with the abilities to communicate with individuals outside one's local tightly-knit social community, a measure of Granovetter's 'strength of weak ties' principle ${ }^{4}$ and to position oneself at particular network locations of high CI that are optimal for information spreading and structural stability of the social network. We note that no causal inference can be established with the present data.

\section{Discussion}

This result highlights the possibility of predicting both financial status and benefits of socially targeted policies based on network metrics, leading to tangible improvements in social marketing campaigns. The high performance of CI among network metrics also suggests the possible role of accessing and mediating information in financial opportunity and well-being 5 . This has an immediate impact in designing optimal marketing campaigns by identifying the affluent targets based on their influential position in a social network. This finding may be also raised to the level of a principle, which would explain the emergence of the phenomenon of CI itself as the result of the optimization of socioeconomic interactions.

\section{Methods}

Code availability. Source code of the CI algorithm is available at the website http://www-levich.engr.ccny.cuny.edu/webpage/hmakse/software-and-data/. Other source code is available on request to the authors.

Data availability. The data sets generated during and/or analysed during the current study are not publicly available for privacy reasons, but are available from the corresponding author on reasonable request.

\section{References}

1. Newman, M. E. The structure and function of complex networks. SIAM Rev. 45, 167-256 (2003).

2. Vespignani, A. \& Caldarelli, G. Large Scale Structure and Dynamics of Complex Networks: from Information Technology to Finance and Natural Science (World Scientific, 2007).

3. Wasserman, S. \& Faust, K. Methods and Applications, Vol. 8 (Cambridge Univ. Press, 1994).

4. Granovetter, M. S. The strength of weak ties. Am. J. Sociol. 78, 1360-1380 (1973).

5. Granovetter, M. The impact of social structure on economic outcomes. J. Econ. Perspect. 19, 33-50 (2005).

6. Eagle, N., Macy, M. \& Claxton, R. Network diversity and economic development. Science 328, 1029-1031 (2010).

7. Singh, V. K., Freeman, L., Lepri, B. \& Pentland, A. S. in 2013 Internation Conference on Social Computing (SocialCom) 174-179 (Washington, DC, USA, 2013).

8. Powell, W. W. \& Smith-Doerr, L. The Handbook of Economic Sociology, Vol. 368, 380 (eds Neil J. Smelser \& Richard Swedberg) (Princeton University Press, Princeton, NJ, USA, 1994).

9. Strang, D. \& Soule, S. A. Diffusion in organizations and social movements: from hybrid corn to poison pills. Annu. Rev. Sociol. 24, 265-290 (1998).

10. Burt, R. S. Structural Holes: the Social Structure of Competition (Harvard Univ. Press, 2009).

11. Page, S. E. The Difference: how the Power of Diversity Creates Better Groups, Firms, Schools, and Societies (Princeton Univ. Press, 2008).

12. Fernandez, R. M. \& Weinberg, N. Getting a job: networks and hiring in a retail bank. Graduate Business School Research Paper No. 1382, 1 (University of Stanford, CA, USA, 1996).

13. Zimmer, C. The Art and Science of Entrepreneurship 3-23 (Ballinger, 1986)

14. Freeman, L. C. Centrality in social networks conceptual clarification. Soc. Networks 1, 215-239 (1978).

15. Toole, J. L. et al. Tracking employment shocks using mobile phone data. J. $R$. Soc. Interface 12, 2015.0185 (2015).

16. Seidel, M.-D. L., Polzer, J. T. \& Stewart, K. J. Friends in high places: the effects of social networks on discrimination in salary negotiations. Admin. Sci. Q. 45, $1-24$ (2000).

17. Cho, E., Myers, S. A. \& Leskovec, J. in Proceedings of the 17th ACM (International Conference on Knowledge Discovery and Data Mining) 1082-1090 (San Diego, CA, USA, 2011).

18. Phithakkitnukoon, S., Smoreda, Z. \& Olivier, P. Socio-geography of human mobility: a study using longitudinal mobile phone data. PLoS ONE 7, e39253 (2012).

19. Deville, P. et al. Scaling identity connects human mobility and social interactions. Proc. Natl Acad. Sci. USA 113, 7047-7052 (2016).

20. Pappalardo, L. et al. An analytical framework to nowcast well-being using mobile phone data. Int. J. Data Sci. Anal. 2, 75-92 (2016).

21. Pan, W., Ghoshal, G., Krumme, C., Cebrian, M. \& Pentland, A. Urban characteristics attributable to density-driven tie formation. Nat. Commun. 4, 1961 (2013). 
22. Gutierrez, T., Krings, G. \& Blondel, V. D. Evaluating socio-economic state of a country analyzing airtime credit and mobile phone datasets. Preprint at https://arxiv.org/abs/1309.4496 (2013).

23. Salah, A. A., Lepri, B., Pianesi, F. \& Pentland, A. S. in International Workshop on Human Behavior Understanding (eds Salah, A. \& Lepri, B.) 1-15 (Springer, 2011).

24. Decuyper, A. et al. Estimating Food Consumption and Poverty Indices with Mobile Phone Data. Technical Report (United Nations Global Pulse, New York, USA, 2014). Preprint at https://arxiv.org/abs/1412.2595 (2014).

25. Blumenstock, J. in Proceedings of 20th ACM SIGKDD (International Conference on Knowledge Discovery and Data Mining) (New York, NY, USA, 2014).

26. Morone, F. \& Makse, H. A. Influence maximization in complex networks through optimal percolation. Nature 524, 65-68 (2015).

27. Stiglitz, J. E. The Price of Inequality: how Today's Divided Society Endangers our Future (W. W. Norton \& Company, 2012).

28. Onnela, J.-P. et al. Structure and tie strengths in mobile communication networks. Proc. Natl Acad. Sci. USA 104, 7332-7336 (2007).

29. Gonzalez, M. C., Hidalgo, C. A. \& Barabasi, A.-L. Understanding individual human mobility patterns. Nature 453, 779-782 (2008).

30. Eagle, N., Pentland, A. S. \& Lazer, D. Inferring friendship network structure by using mobile phone data. Proc. Natl Acad. Sci. USA 106, 15274-15278 (2009).

31. Pei, S. \& Makse, H. A. Spreading dynamics in complex networks. J. Stat. Mech. Theor. Exp. 2013, P12002 (2013).

32. Page, L., Brin, S., Motwani, R. \& Winograd, T. The Pagerank Citation Ranking: bringing Order to the Web. Technical Report 422 (Stanford InfoLab, Palo Alto, CA, USA, 1998).

33. Kitsak, M. et al. Identification of inuential spreaders in complex networks. Nat. Phys. 6, 888-893 (2010).

34. Kempe, D., Kleinberg, J. \& Tardos, É. in Proceedings of 9th ACM SIGKDD (International Conference on Knowledge Discovery and Data Mining) 137-146 (Seattle, WA, USA, 2003).

35. Wildt, A. R. \& Ahtola, O. Analysis of Covariance, Vol. 12 (Sage Publications, 1978).

36. Blondel, V. D., Guillaume, J.-L., Lambiotte, R. \& Lefebvre, E. Fast unfolding of communities in large networks. J. Stat. Mech. Theor. Exp. 2008, P10008 (2008).

37. Newman, M. E. Analysis of weighted networks. Phys. Rev. E 70, 056131 (2004).

\section{Acknowledgements}

This work was funded by ARL Cooperative Agreement Number W911NF-09-2-0053 (the ARL Network Science CTA), NIH-NIGMS 1R21GM107641, NIH-NIBIB 1R01EB022720 NSF IIS-1515022 and NSF-PoLS PHY-1305476. We thank B. Min for discussions.

\section{Author contributions}

H.A.M. directed the project. S.L. developed the data and network analysis. F.M. performed the data validation and provided the support on network theory. C.S. and M.T. collected and processed all original data. All authors contributed in writing the paper.

\section{Additional information}

Supplementary Information accompanies this paper at http://www.nature.com/ naturecommunications

Competing interests: The authors declare no competing financial interests.

Reprints and permission information is available online at http://npg.nature.com/ reprintsandpermissions/

How to cite this article: Luo, S. et al. Inferring personal economic status from social network location. Nat. Commun. 8, 15227 doi: 10.1038/ncomms15227 (2017).

Publisher's note: Springer Nature remains neutral with regard to jurisdictional claims in published maps and institutional affiliations.

(c) (i) This work is licensed under a Creative Commons Attribution 4.0 cc) International License. The images or other third party material in this article are included in the article's Creative Commons license, unless indicated otherwise in the credit line; if the material is not included under the Creative Commons license, users will need to obtain permission from the license holder to reproduce the material. To view a copy of this license, visit http://creativecommons.org/licenses/by/4.0/

(C) The Author(s) 2017 